\title{
MULTICOLOR IMAGES OF SPATIALLY RESOLVED STRUCTURES AROUND HIGH-REDSHIFT QUASARS ${ }^{1}$
}

\author{
MATTHEW D. LEHNERT ${ }^{2}$ AND TIMOTHY M. HECKMAN ${ }^{2}$ \\ Space Telescope Science Institute; and Rowland Department of Physics and Astronomy, The Johns Hopkins University, Baltimore, MD 21218 \\ KenNeth C. Chambers ${ }^{2}$ \\ Institute for Astronomy, University of Hawaii, Honolulu, HI 96822 \\ AND \\ GeORGE K. MiLEY ${ }^{2,3}$ \\ Sterrewacht, Leiden, Postbus 9513, 2300RA Leiden, The Netherlands \\ Received 1991 June 29; accepted 1992 January 7
}

\begin{abstract}
We present multicolor, optical, and near-IR imaging of spatially resolved continuum-emitting structures ("fuzz") around six high-redshift $(z=2-3)$ radio-loud quasars selected from the earlier imaging survey of Heckman et al. We argue that this fuzz is most plausibly interpreted as the stellar population of the host galaxy of the quasar. All six quasars are well resolved in the near-IR images (corresponding to emission at $\approx 6000$ to $7000 \AA$ in the quasar rest frame). The implied $K$-corrected absolute visual magnitudes for the fuzz are -24 to -26 (for $H_{0}=75 \mathrm{~km} \mathrm{~s}^{-1} \mathrm{Mpc}^{-1}$ and $q_{0}=0$ ). This is several magnitudes brighter than the most luminous galaxies in the present-day universe (namely, the "first-ranked" giant ellipticals in rich clusters and host galaxies of low- $z$ radio-loud quasars). In four of the six cases the fuzz is at least 1.5 mag redder in $(B-K)$ than the central quasar, so that it is unlikely that the near-IR fuzz is generally quasar light scattered by either dust or electrons in the quasar halo. In two cases, the fuzz is well resolved in the optical images $(\approx 1400 \AA$ in the QSR rest frame), in two other cases the optical fuzz is possibly resolved and in the last two cases the optical fuzz is unresolved. The measured spectral energy distributions in the two best-resolved cases are similar to those of the most actively star-forming present-day galaxies (e.g., Magellanic Irregular and Markarian starburst galaxies). If the rest-frame UV fuzz is indeed starlight, the fuzz luminosities imply starformation rates of several hundred solar masses per year in these two objects. The apparent $K$ magnitudes for the fuzz lie along the remarkably tight $K$ versus redshift "IR Hubble diagram" for radio galaxies discovered by Lilly in 1989. The available data are also consistent with the fuzz around high-z radio-loud quasars having spectral energy distributions that are similar to those of high-z radio galaxies. These data imply that radioloud quasars and radio galaxies at high redshift are intimately related phenomena and are consistent with some recent efforts to "unify" the two AGN classes.
\end{abstract}

Subject headings: galaxies: stellar content - quasars: general — radio continuum: galaxies

\section{INTRODUCTION}

The study of AGNs at high redshifts offers a unique opportunity to investigate conditions in the early universe. Probing the environments of such objects is essential if we are to understand the processes that drove the strong cosmic evolution of the AGN phenomenon (e.g., Hartwick \& Schade 1990) and unravel the relationship of high-redshift AGNs to the formation and early evolution of galaxies (e.g., Rees 1988; Carlberg 1990; Turner 1991).

Recently, there has been much effort given to deep, multicolor optical, and near-IR imaging of high-redshift $(z>1)$ radio galaxies (e.g., Chambers \& Miley 1990; Lilly 1990). Such observations have discovered remarkable properties for these galaxies. They have enormous $(\approx 100 \mathrm{kpc}$ scale $)$ emission-line and continuum structures that preferentially align with the radio source axis (Chambers, Miley, \& van Breugel 1987; McCarthy et al. 1987). If the continuum emission is due to the

\footnotetext{
${ }^{1}$ Based partially on data taken at the United Kingdom Infrared Telescope.

2 Visiting observers at the Kitt Peak National Observatory and Cerro Tololo Inter-American Observatory of the National Optical Astronomy Observatories, operated by AURA under contract with the National Science Foundation.

${ }^{3}$ Visiting Astronomer at the Space Telescope Science Institute.
}

stellar population of the radio galaxy, then the most natural interpretation is that the radio source has triggered the formation of a galaxy's worth of stars at a rate of several hundred $M_{\odot}$ per year (e.g., Chambers \& Charlot 1990; Begelman \& Cioffi 1989; Rees 1989). Moreover, the tight linear relationship between the apparent $K$-magnitude and the redshift for radio galaxies strongly suggests that these objects represent the most massive galaxies in the universe at any given epoch (Lilly 1989, 1990). On the other hand, the recent discovery that the spatially extended continuum emission from at least one high- $z$ radio galaxy is polarized (di Serego Alighieri et al. 1989; Scarrott, Rolph, \& Tadhunter 1990) suggests that scattering of the light from a "hidden" AGN by dust or electrons along the radio axis may make a significant contribution to the aligned continuum emission (see also Fabian 1989).

Expanding the investigation of the environments of high- $z$ AGNs to other AGN classes is therefore important both with regard to the broad issues discussed in the first paragraph and the more specific ones in the second. Radio-loud quasars (hereafter "QSRs") are particularly appropriate subjects for such investigation for a variety of reasons. Several types of "unification schemes" have been proposed that relate the QSRs and radio galaxies through viewing angle, evolutionary, 
and environmental effects (e.g., Barthel 1989; Norman \& Miley 1984; Neff \& Hutchings 1990). Comparisons of the surroundings of high-z QSRs and radio galaxies allow us to test these ideas. Moderately large samples of QSRs already exist with good radio maps and optical spectroscopy (e.g., Barthel et al. 1988; Barthel, Tytler, \& Thompson 1990). The extensive data on the environments of similar objects at low redshifts (e.g., Smith et al. 1986; Crawford, Fabian, \& Johnstone 1988; Smith \& Heckman 1990; Ellingson, Green \& Yee 1991) can be used to make direct comparisons to the high-redshift QSRs to search for evidence for evolution in the QSR environment with cosmic time.

In two recent papers (Heckman et al. 1991a [hereafter HLVM], 1991b) we have established that high- $z(z \approx 2$ to 3$)$ QSRs are often immersed in galaxy-scale emission-line and UV-continuum "fuzz" whose properties are broadly similar to the structures associated with the high- $z$ radio galaxies. In the present paper, we report the results of multiwaveband continuum imaging of six of these QSRs. These observations were undertaken to address several specific questions.

How does the spectral energy distribution ("SED") of the QSR fuzz compare to that of the central QSR itself? Fuzz produced by scattering of QSR light by electrons or dust grains in the QSR halo should be at least as blue as the QSR. On the other hand, if the QSR fuzz has an SED similar to that of the high-z radio galaxies or of actively star-forming (late Hubbletype) galaxies in the present-day universe, it should be significantly redder than the QSR. Images at $K$-band combined with optical images (corresponding to an approximate wavelength range from 1300 to $6500 \AA$ in the QSR rest frame) allow us to make such a comparison.

How similar is the SED of the QSR fuzz in both shape and luminosity to the SED of high-z radio galaxies? In particular, does the QSR fuzz lie along the tight $K$ versus $z$ relation defined by the radio galaxies? Can we see evidence for alignment of the radio and rest-frame UV/optical continuum axes in the QSRs?

If the QSR fuzz is indeed starlight, what inferences can we make about the stellar population and the history of recent star formation in the QSR host galaxies?

\section{OBSERVATIONS AND DATA REDUCTION}

The six objects studied here are a small subset of the objects studied by Barthel et al. (1988), Barthel, Tytler, \& Thompson (1990), and HLVM. Extensive discussion of the radio and optical properties of the QSRs can be found in these papers. All six QSRs in the present paper have strong radio emission on arcsecond or larger scales and redshifts of 2-3. They are representative of that half of the QSRs studied by HLVM that were found to be either definitely or possibly resolved in their optical continuum images. Our observations of these QSRs are summarized in Table 1.

\subsection{Near-IR Observations}

The $K$-band $(2.2 \mu \mathrm{m})$ image of Q0445+ 019 was taken on 1990 September 24 using the IRCAM imager on the $3.5 \mathrm{~m}$ United Kingdom Infrared Telescope (UKIRT). IRCAM uses a $58 \times 62$ InSb focal plane array and camera with 0.6 pixel $^{-1}$ (McLean 1988). To improve the quality of the flat-fielding (and thereby increase the dynamic range of the image) we took many exposures, moving the telescope between exposures to place the QSR on different regions of the detector. An eightposition raster cycle was adopted, reading out the chip every $50 \mathrm{~s}$ and spending $250 \mathrm{~s}$ at each position. We completed four such raster cycles, resulting in a total integration time of $8000 \mathrm{~s}$. The flat-field and sky frames were constructed by median filtering the unregistered raster sets. After flat-fielding and background subtraction, it was necessary to shift the individual frames so that they were registered and then added together. Registration was accomplished by centering on a star visible in individual frames. Flus calibration was done via observations of the standard stars HD 18881, GL 748, and GL 105.5 (Elias et al. 1982).

The $K$-band IR images for the other five QSRs and the $J$-band $(1.2 \mu \mathrm{m})$ image of Q0805+097 were obtained on the nights of 1991 March 29-31 at Kitt Peak National Observatory (KPNO) using the $4 \mathrm{~m}$ telescope and the IRIM detector. IRIM also uses a $58 \times 62 \mathrm{InSb}$ array, and with the $\mathrm{f} / 8$ infrared secondary yields 0.4 pixel $^{-1}$. A nine-position raster pattern with $90 \mathrm{~s}$ exposure times similar to the UKIRT observing scheme

TABLE 1

OBSERVATIONS

\begin{tabular}{|c|c|c|c|c|c|c|c|}
\hline $\begin{array}{l}\text { QSR } \\
\text { (1) }\end{array}$ & $\begin{array}{l}\text { Filter } \\
\text { (2) }\end{array}$ & $\begin{array}{l}\text { Telescope } \\
\text { (3) }\end{array}$ & $\begin{array}{l}\text { Detector } \\
\text { (4) }\end{array}$ & $\begin{array}{l}\text { Integration } \\
\text { (5) }\end{array}$ & $\begin{array}{l}\text { Pixel } \\
\text { (6) }\end{array}$ & $\begin{array}{c}\text { PSF } \\
\text { (7) }\end{array}$ & $\begin{array}{c}\text { Dates } \\
(8)\end{array}$ \\
\hline \multirow[t]{4}{*}{$\mathrm{Q} 0445+097 \ldots \ldots \ldots$} & $U$ & KPNO $4 \mathrm{~m}$ & PFCCD/T12 & 600 & 0.3 & 1.0 & 1988 Nov \\
\hline & $B$ & CTIO $4 \mathrm{~m}$ & PFCCD/TI2 & 900 & 0.3 & 1.6 & $1990 \mathrm{Dec}$ \\
\hline & $\boldsymbol{R}$ & CTI0 $4 \mathrm{~m}$ & PFCCD/TI2 & 900 & 0.3 & 1.4 & $1990 \mathrm{Dec}$ \\
\hline & $K$ & UKIRT & IRCAM & 8000 & 0.6 & 1.5 & $1990 \mathrm{Sep}$ \\
\hline \multirow[t]{2}{*}{$\mathrm{Q} 0730+257 \ldots \ldots \ldots$} & $B$ & KPNO $4 \mathrm{~m}$ & PFCCD/TI2 & 600 & 0.3 & 1.8 & 1988 Nov \\
\hline & $K$ & KPNO $4 \mathrm{~m}$ & IRIM & 8100 & 0.4 & 1.3 & $1991 \mathrm{Mar}$ \\
\hline \multirow[t]{3}{*}{$\mathrm{Q} 0805+046 \ldots \ldots \ldots$} & $B$ & KPNO 4 m & PFCCD/T12 & 600 & 0.3 & 1.2 & 1988 Nov \\
\hline & $J$ & KPNO $4 \mathrm{~m}$ & IRIM & 6480 & 0.4 & 1.3 & $1991 \mathrm{Mar}$ \\
\hline & $K$ & KPNO $4 \mathrm{~m}$ & IRIM & 8100 & 0.4 & 1.3 & $1991 \mathrm{Mar}$ \\
\hline \multirow[t]{2}{*}{ 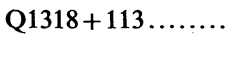 } & $B$ & KPNO $4 \mathrm{~m}$ & PFCCD/TI2 & 640 & 0.3 & 1.4 & 1988 May \\
\hline & $K$ & KPNO $4 \mathrm{~m}$ & IRIM & 8100 & 0.4 & 1.2 & $1991 \mathrm{Mar}$ \\
\hline \multirow{2}{*}{ Q1354+258 ....... } & $B$ & KPNO $4 \mathrm{~m}$ & PFCCD/TI2 & 512 & 0.3 & 1.4 & 1988 May \\
\hline & $K$ & KPNO $4 \mathrm{~m}$ & IRIM & 7290 & 0.4 & 1.2 & $1991 \mathrm{Mar}$ \\
\hline \multirow[t]{2}{*}{ 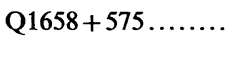 } & $B$ & KPNO $4 \mathrm{~m}$ & PFCCD/T12 & 512 & 0.3 & 1.3 & 1988 May \\
\hline & $K$ & KPNO 4 m & IRIM & 8910 & 0.4 & 1.2 & $1991 \mathrm{Mar}$ \\
\hline
\end{tabular}

CoL. (5).--Total integration time in seconds.

Col. (6).- - Pixel sizes in arcseconds.

CoL. (7). - The full width at half-maximum intensity of the point-spread-function of the image as determined from observations of stars (see text). 
was adopted. After one or two sets of nine exposures on the QSR, the telescope was offset to a star located within a few arcminutes of the QSR and the raster pattern was repeated with $10 \mathrm{~s}$ integration times. This procedure was followed to accurately measure the point-spread function (PSF) of the images. The total number of raster patterns obtained per QSR varied from 8 to 11 , and total integration times therefore ranged from 6480 to $8910 \mathrm{~s}$ (see Table 1 for details). The flats and sky frames were constructed by median filtering all the unregistered frames of each raster cycle. Because the QSRs were too faint to be visible in the individual exposures, registration was accomplished by using the nominal offsets input to the telescope for the raster pattern. Tests on bright stars showed that the nominal offsets match the actual offsets to within 0 ". 1 ( $<$ few tenths of a pixel) and do not significantly affect the PSF of the final image. We emphasize that the QSR and star (PSF) rasters were processed in exactly the same way. Finally, we tested the linearity of the array by taking dome flats with signals spanning the range from nearly zero counts to over twice the maximum amount in any of our observations. No detectable nonlinearity was found. Flux calibration of these images was done with observations of Elias et al. (1982) standards.

\subsection{Optical Images}

Most of the optical images discussed in this paper have been previously published in HLVM. New $B$ - and $R$-band CCD images of Q0445+097 were obtained on the night of 1990 December 21 at Cerro Tololo Inter-American Observatory (CTIO) using the $4 \mathrm{~m}$ telescope and the Prime-Focus CCD imaging system with the TI2 CCD. The pixel size is 0.30 , and both images had integration times of $900 \mathrm{~s}$. The images were flat-fielded using a set of median-filtered images taken of the twilight sky and were flux-calibrated using E-region standard stars (Graham 1982) taken at the beginning and end of the night.

\subsection{Image Analysis}

In order to demonstrate that the QSRs are spatially resolved, and to determine the amount of resolved flux, it is crucial to determine accurately the PSF characterizing each image. We have determined the PSF in three different ways for the optical images, the UKIRT IR image, and the KPNO IR images.

For the optical images, we have used the brightest unsaturated stars (typically five to eight per frame) to both measure the PSF and assess its variation across the imaged field. These variations are due primarily to focus variations across the wrinkled surfaces of the TI CCDs used in the fast $\mathrm{f} / 2.8$ beams at the prime foci of the KPNO and CTIO $4 \mathrm{~m}$ telescopes. From our analysis of an ensemble of $\approx 50$ stars in the eight optical frames, we find that the fluctuations in the PSF shape across the entire chip introduce $1 \sigma$ uncertainties in the flux of a 5"-10" scale spatially resolved structure surrounding a QSR of $\approx 2 \%$ of the central QSR flux. This uncertainty can be cut in half if only stars located within $\sim 30^{\prime \prime}$ of the QSR are used to specify the PSF. We have done this whenever possible for all the QSRs discussed in HLVM.

For the UKIRT IR image of Q0445+097, the PSF was determined from observations of four different stars observed before and after the set of QSR observations. The PSFs of these four stars are indistinguishable from one another, and we have therefore constructed a PSF from their flux-weighted average. As already discussed, the procedure adopted for the
IR imaging at KPNO consisted of many series of interleaved star and QSR observations. The PSF was therefore measured nearly contemporaneously with the QSR images and sampled exactly the same regions on the detector. Because the QSR integration times at KPNO were longer than the integration times on the PSF reference stars ( $90 \mathrm{~s}$ vs. $10 \mathrm{~s}$ ), it is possible, in principle, that image drift might artificially broaden the PSF in the QSR frames. Tests on stars using varying exposure times imply that this should not be a problem, however.

Having determined the PSF, we performed two kinds of analyses to determine whether the QSRs were spatially resolved. Following the procedure in HLVM, we first compared the azimuthal average of the QSR radial surface brightness profile to that of the PSF. This comparison is made graphically in Figure 1 for all the optical and near-IR images of all six of the QSRs. The second method was to register the full twodimensional PSF and QSR images and then scale the PSF image so that the QSR and the PSF had the same total counts within a small box (area $\approx 2 \operatorname{arcsec}{ }^{\overline{2}}$ ) centered on the peak. This scaled PSF was then subtracted off the QSR, providing us with both an indication of the morphology of the material near the peak and an estimate of the spatially resolved flux. This latter is strictly speaking a lower limit to the true fuzz flux since it explicitly assumes that the fuzz has zero flux within the small central box. We will show below the QSR fuzz is remarkably similar to the high-redshift radio galaxies. Analysis of the optical and near-IR images of radio galaxies at similar redshifts show that exclusion of this central-most light would typically dim the radio galaxies by only several tenths of a magnitude. Since this is less than the other uncertainties in the fuzz magnitudes, we have not attempted to correct the derived QSR fuzz properties for this effect.

\section{RESULTS}

All of the available near-IR and optical images of the six QSRs are shown in contour plots in Figure 2 and the parameters derived from these images are given in Table 2 . We have images in four colors for $\mathrm{Q} 0445+097(U, B, R$, and $K)$, in three colors for $\mathrm{Q} 0805+046(B, J$, and $K)$, and only two colors $(B$ and $K$ ) for the other four QSRs.

Each of the six QSRs is clearly resolved in the near-IR images with the spatially resolved material contributing $\approx 20 \% \pm 5 \%$ of the total flux. The optical images of Q0445+ 097 and Q1318+113 are well resolved (since the fuzz contributes from $15 \%$ to $38 \%$ of the total flux) while $\mathrm{Q} 0730+257, \mathrm{Q} 0805+046, \mathrm{Q} 1354+258$, and $\mathrm{Q} 1658+575$ must be considered only marginally resolved or unresolved in the optical (since the fuzz provides only $\sim 3 \%-6 \%$ of the total flux). In the cases of Q1354+258 and Q1658+575, the morphological similarity between the structures visible in the $B$ and $K$-band images (see below) give us some confidence that the QSRs are probably weakly resolved in the $B$ frames. We have adopted the flux values for the fuzz derived by HLVM for these two QSRs, but emphasize that the values are uncertain by a factor of 2 . The $B$-band fuzz in Q0805+046 is strongly contaminated by $\mathrm{Ly} \alpha$ line emission, while in the Q0730+257 image the seeing was poor in our optical frame $\left(\approx 2^{\prime \prime} \mathrm{FWHM}\right)$. We have adopted upper limits of $4 \%$ of the QSR flux for the optical continuum fuzz in these two cases. The apparent magnitudes of the spatially resolved material range from $\approx 21$ to 23 in the optical and from $\approx 17$ to 19 in the near-IR.

We have used the Ly $\alpha$ images in HLVM to estimate the contamination of the optical continuum fuzz by the spatially 

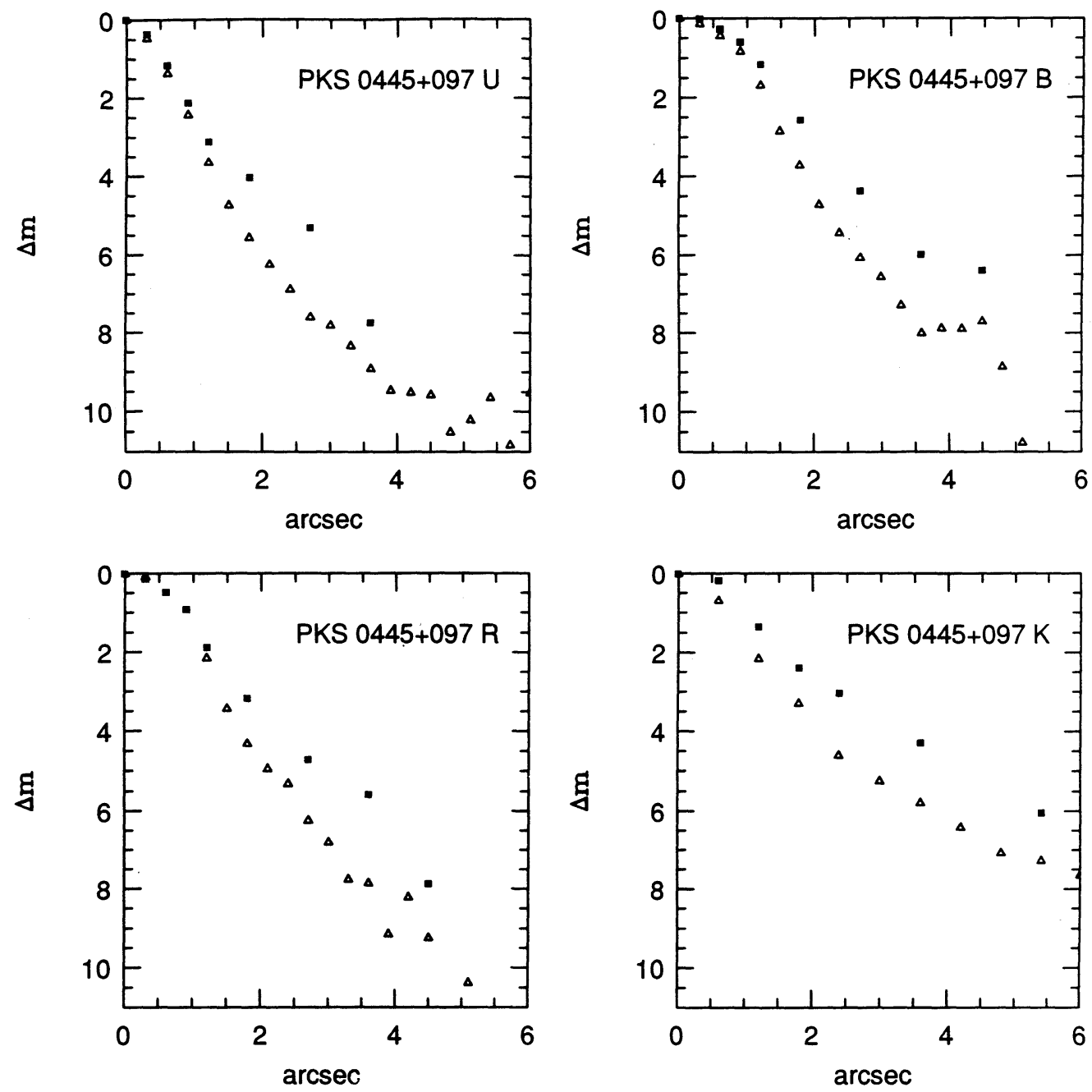

Fig. 1.-Comparison of the azimuthally averaged radial surface brightness profiles (RSBPs) of 15 multicolor images of our sample of 6 QSRs (solid boxes) to the corresponding point spread functions (PSFs) as measured with bright stars (open triangles). The surface brightnesses are given in magnitudes relative to the brightest central pixel.

extended emission-line gas. The spectra of the fuzz published in Heckman et al. (1991b) show that any other UV emission lines are negligible in strength $(<10 \%$ of $L y \alpha)$. Apart from $\mathrm{Q} 0805+046$ (which is unresolved in the $B$-band image in any case) Ly $\alpha$ emission produces only $\approx 12 \%$ on-average of the spatially resolved optical broad-band emission for the QSRs (see Table 2 for details). Corrections for emission lines in the spatially extended $K$-band fuzz are more problematic, though in most cases the $\mathrm{H} \alpha$ line is redshifted into the wings of the $K$ filter bandpass. The morphology of the spatially resolved structures in Ly $\alpha$ (see HLVM) and $K$-band images are generally sufficiently different from one another that it is unlikely that emission-lines make a dominant contribution to the $K$-band fuzz. More quantitatively, McCarthy, Elston, \& Eisenhardt (1992) have obtained near-IR spectra of two high- $z$ radio galaxies and find that the flux of the $\mathrm{H} \alpha+[\mathrm{N} \mathrm{II}] \lambda \lambda 6548,6584$ blend is $\approx 20-30 \%$ that of Ly $\alpha$. If the QSR nebulae have similar emission-line spectra to the nebulae associated with high $z$ radio galaxies (as appears to be the case in the UV-see Heckman et al. 1991b), then the fluxes of the lines likely to contaminate the $K$-band "fuzz" (chiefly the $\mathrm{H} \alpha,[\mathrm{N}$ II $] \lambda \lambda 6548$, 6584 , and [S II] $\lambda \lambda 6716,6731$ lines) will be $\sim 30 \%$ of Ly $\alpha$. In this case, emission-line contamination of the fuzz at $K$ is negligible $(\leq 3 \%)$ except in Q1318+113 $(13 \%)$. See Table 2 for the specific values.

Typical sizes of the fuzz measured at isophotal levels of $B \approx 27$ and $K \approx 22 \mathrm{mag}$ per square arcsecond $(\approx 1 \%$ and approximately a few hundredths of a percent of the night sky, respectively) are $6 "-10^{\prime \prime}$. While the amount of morphological information is clearly limited, the fuzz is quite irregular and asymmetric in most of the QSRs.

In the cases of Q0445+ 097 and Q1318+113 (which are well resolved in the optical) the optical and near-IR morphologies are strikingly similar over a factor of 5-6 in wavelength. The strongly asymmetric "plume" extending $\approx 4$ arcsec to the ESE of Q0445 + 097 is visible in all four frames, but a fainter feature extending $\approx 6^{\prime \prime}$ to the SSW is visible only at $U$ and may be spurious. The brightest structure in the optical and near-IR images of Q1318 + 113 is an $\approx 4$ " long plume to $\mathrm{E}$, but a fainter feature $\approx 4$ " to the SW and the detached patch of emission $\approx 7$ " to the NW of the QSR are also present in both the $B$ and $K$ frames. The SW plume may be significantly contaminated by emission lines in both images, since there is strong Ly $\alpha$ emission out to a radius of $\approx 9^{\prime \prime}$ to the SSW of the QSR (see 

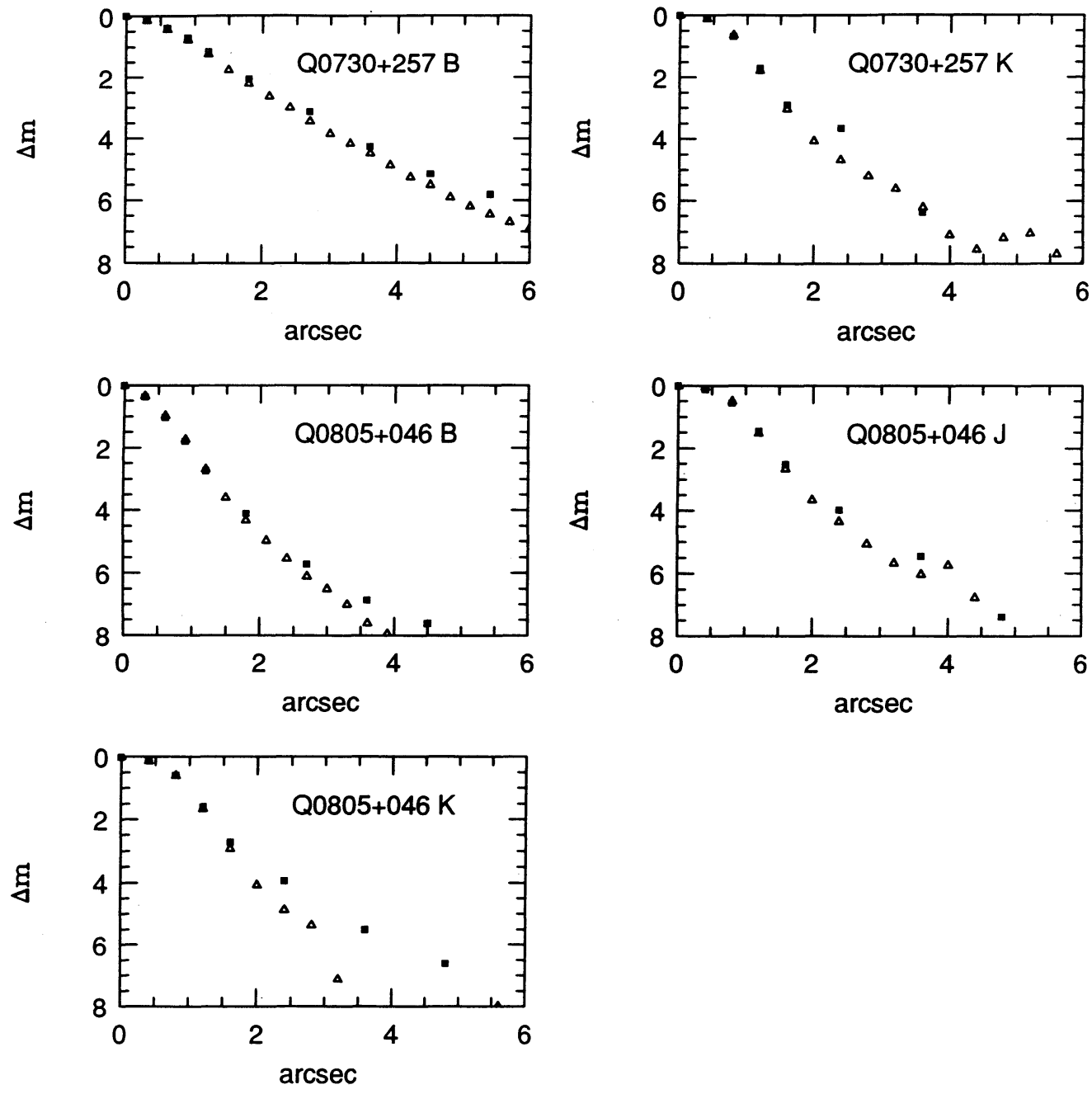

Fig. 1.-continued

HLVM). Even Q1354+258 and Q1658 + 575, which are only very marginally resolved at best in the respective optical images, have similar morphologies in the $B$ and $K$ images. A plume extending $5^{\prime \prime}-6$ " NNE of Q1354 +258 is visible in the $B$ and $K$ frames. The morphology of Q $1658+575$ is particularly interesting. In the $\mathrm{K}$ image the QSR appears to be "joined" by a curved and patchy bridge of emission to a fainter compact object (with $K=17.0$ and $B=22.2$ ) located some $9^{\prime \prime}$ to the SSW. Hints of this bridge feature are visible to the SSW of the QSR in the B image as well. Spectroscopy of the SSW knot will be required to establish its relationship (if any) to the QSR. It does not show up as a detectable Ly $\alpha$ emission-line source at the QSR redshift in the narrow-band images of HLVM. Another intriguing structure consists of two knots embeded in a more diffuse linear structure $\approx 7$ " to the SSE of Q0805 + 046 . This is clearly visible in the $B, J$, and $K$ images, with the entire structure having an apparent magnitude of 22.8, 19.3, and 18.2 in the three respective bands. This structure is engulfed in the outermost detectable portion of the Ly $\alpha$ nebula associated with this QSR (see HLVM).

As an attempt to roughly parameterize the colors of the spatially extended fuzz and compare these to the colors of the central QSR, we have defined an effective spectral index between $B(0.44 \mu \mathrm{m})$ and $K(2.2 \mu \mathrm{m})$ as $\alpha_{K}^{B} \equiv-\log \left(S_{v}^{B} / S_{v}^{K}\right) / \log$
$\left(v_{B} / v_{K}\right)$. Our calculated values for $\alpha_{K}^{B}$ (corrected for the effects of Galactic reddening) are listed in Table 2 for both the QSRs and the fuzz. Note that we have not corrected $\alpha_{K}^{B}$ for the effects of emission-lines in the $B$ and $K$ bandpasses (these corrections to $\alpha_{K}^{B}$ are always $\leq 0.1$ in any case). Excluding the unusually red object Q0445+097, the mean value of $\alpha_{K}^{B}$ we derived for the central QSR $(\sim 0.3)$ is consistent with the mean SED derived for large samples of QSRs and radio-quiet quasars by Sanders et al. (1989) and Francis et al. (1991). The most interesting result is that the spatially resolved fuzz is significantly redder than the QSR, in four of the six cases: Q0445+097 and Q1318+113 have similar fuzz and QSR colors, but the fuzz is at least 1.5 mag redder than the QSR in $(B-K)$ in the other four cases.

\section{DISCUSSION}

\subsection{Comparison to High-Redshift Radio Galaxies}

The relationship between QSRs and powerful radio galaxies is an issue of considerable interest, especially in light of efforts to "unify" these two classes through differences in viewing angle, environment, or evolutionary state (e.g., Barthel 1989; Norman \& Miley 1984; Neff \& Hutchings 1990). In particular, the "viewing angle" scheme of Barthel (in which QSRs and 

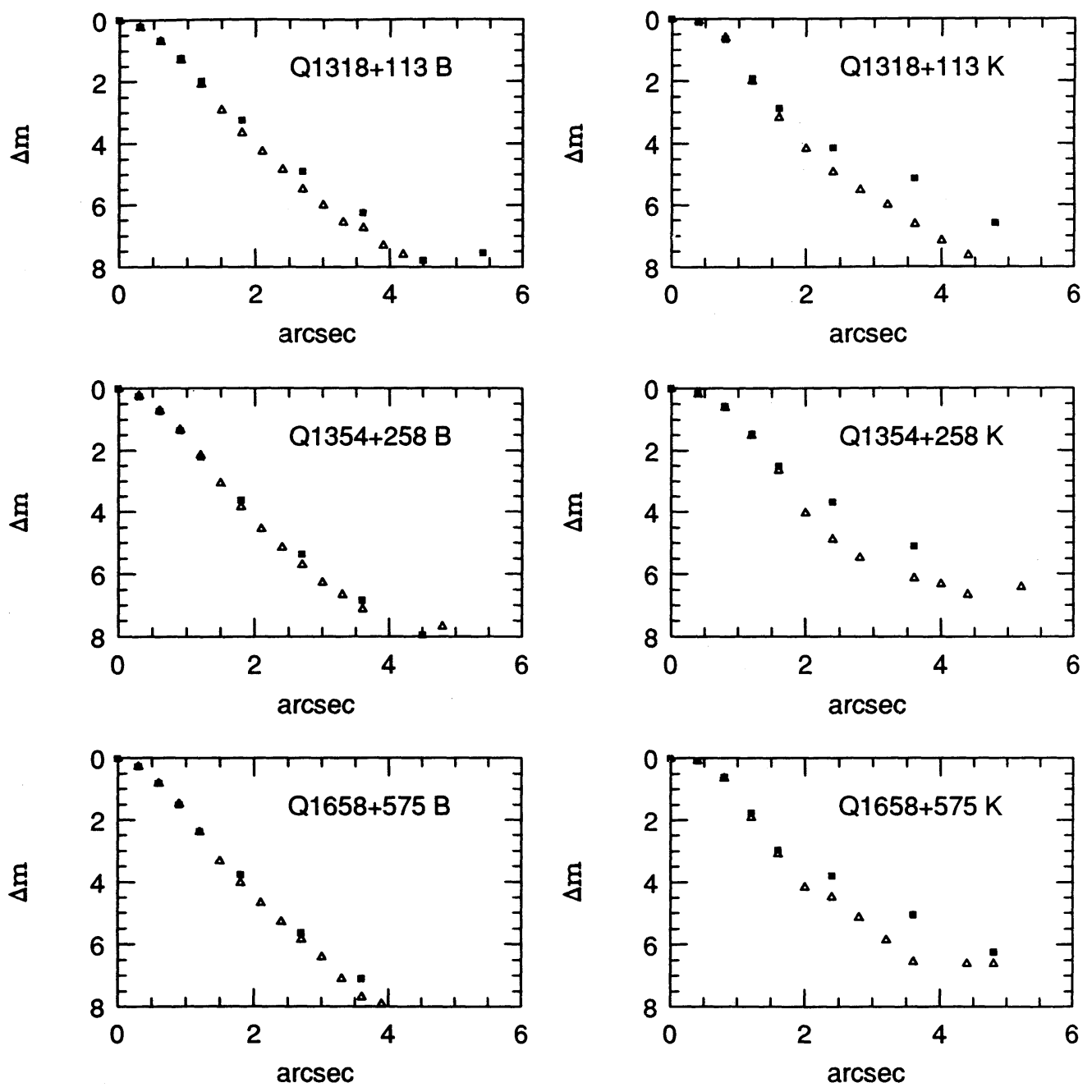

Fig. 1.-continued

radio galaxies are drawn from the same parent population but are viewed preferentially at small or large angles, respectively, to the radio axis) predicts that the luminosity and color of the QSR fuzz should be very similar (if not identical) to those of radio galaxies of similar redshifts and radio powers.

In Figure 3 we have plotted the apparent $K$ magnitudes of the fuzz of our six QSRs on the "IR Hubble Diagram " $(K$ vs. $z)$ defined by radio galaxies (as determined from published radio galaxy data). Given the uncertainties in the $K$ magnitudes for the QSR fuzz (typically \pm 0.4 mag not including the uncertain correction for the "missing" central light) the agreement between the QSRs and radio galaxies at $z=2-3$ is remarkably good. Since there are presently data for only a handful of objects in this redshift range, a more detailed comparison of the QSRs and radio galaxies is not yet possible. In particular, the possible trend for the QSR hosts to be slightly brighter than the radio galaxies in Figure 3 needs to be confirmed (see Hutchings 1987).

The optical-to-near-IR colors of the best-resolved QSR fuzz $(Q 0445+097$ and Q1318+113) appear to be similar to the published SEDs of the bluer of the high-redshift radio galaxies -e.g., 3C 239 and $1204+35$ in Lilly (1989), 3C 266, 3C 368, and 4C 41.17 in Chambers \& Charlot (1990), MRC
2025-218 in McCarthy (1991), and 53W002 in Windhorst et al. (1991). The blueness of the QSR fuzz in these two cases is likely to be a selection effect since these are among the QSRs in HLVM with the brightest optical fuzz. However, we know of no radio galaxy quite as blue as the fuzz of $Q 1318+113$. A future comparison of larger, unbiased samples of QSRs and radio galaxies with well-matched distributions in redshift and radio power would be interesting in this regard.

The "alignment effect" between the radio and the optical/ near-IR continuum axes is still the most puzzling aspect of the high- $z$ radio galaxy phenomenon. In our earlier paper (HLVM) we were able to reliably determine a major axis position angle for the optical continuum fuzz for only four high-z QSRs (Q0225-014, Q0445+097, Q0549-213, and $\mathrm{Q} 1318+113)$. We found no evidence in this very small sample for an alignment effect. The new IR images presented in this paper allow us to add Q0730 + 257, Q0805 + 046, Q1354+258, and Q1658 + 575 to the sample. In this expanded sample, we see a weak tendency for an alignment effect; five of the eight QSRs have an optical and/or near-IR continuum axis that aligns to better than $30^{\circ}$ with the radio axis. We emphasize that this result is not statistically significant and must be checked with a much larger sample of QSRs. 

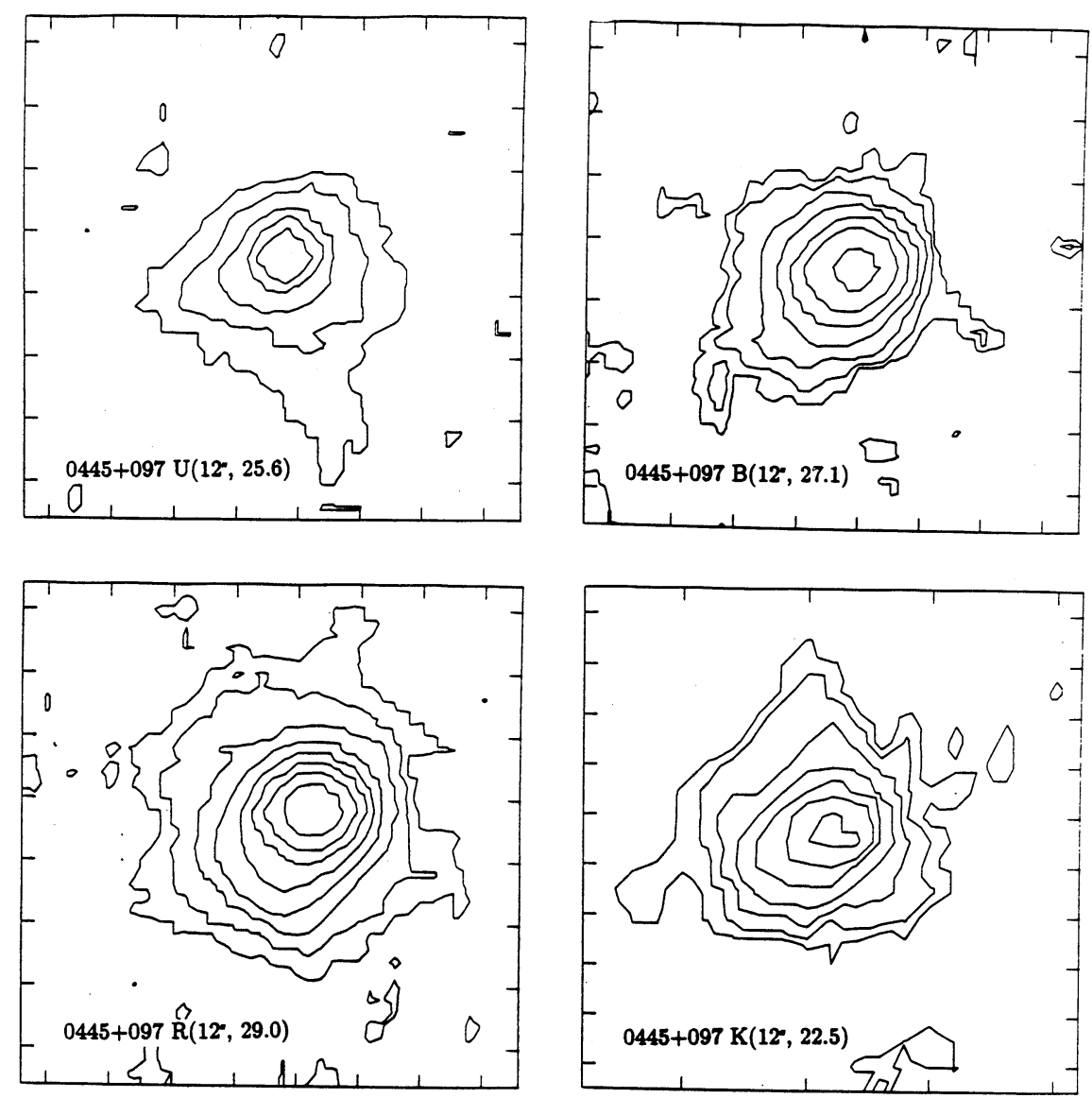

FIG. 2. - Contour plots of the 15 multicolor images of the si $\alpha$ QSRs in our sample. All the images have been median-filtered with a 1 ".2 2 1".2 box to remove cosmic rays and emphasize faint structures. The sizes of the displayed field and the values of the lowest contour levels (in magnitudes arcsec ${ }^{-2}$ ) are given in each plot. Each successive contour represents a factor of 2 increase in surface brightness $\left(\approx 0.75 \mathrm{mag} \operatorname{arcsec}^{-2}\right)$. We emphasize that the light from the central unresolved QSR has not been subtracted from these images and typically dominates the light within a radius of $\approx 2$ " of the center (see Fig. 1 for quantitative details).

\subsection{Origin of the Fuzz Light}

The origin of the optical and near-IR continuum light in the high-redshift radio galaxies has remained controversial. Given the similarity of the QSR fuzz and the high- $z$ radio galaxies (see above), it is useful to examine our QSR data from the perspectives of each of the three basic types of models advanced for the radio galaxies: (1) starlight from a foreground galaxy (e.g., LeFevre \& Hammer 1988), (2) light from a central QSR emitted anisotropically along the radio axis and then scattered by dust or electrons in the halo of the galaxy (e.g., Fabian 1989; di Serego Alighieri et al. 1989; Scarrott, Rolph, \& Tadhunter 1990), and (3) starlight from a population of either young stars alone (e.g., Chambers \& Charlot 1990) or a mix of old and very young stars (e.g., Lilly 1989).

In four of the six QSRs in our sample there is independent evidence for intervening matter in the form of one or more detectable $\mathrm{Mg}$ II $\lambda 2798$ doublet absorption lines at $z \approx 1$ (Barthel, Tytler, \& Thompson 1990; see Table 3). These lines are presumably produced by gas in the halo of an intervening galaxy (e.g., Bergeron \& Boisse 1991). Despite this, we believe there are at least three good reasons why an intervening galaxy is not likely to be responsible for the bulk of the fuzz we have detected.

We have calculated the absolute $R$ magnitudes for the fuzz under the assumption that they are at the redshifts of the $\mathrm{Mg}_{\text {II }}$ absorbers. We have done this using the available optical and
near-IR images to estimate the fuzz flux density at the wavelength corresponding to the center of the $R$ filter at the redshift of the absorption $\left[\lambda=\left(1+z_{\mathrm{abs}}\right) 6500 \AA\right]$. The results are listed in Table 3, where it can be seen that the fuzz would have $M_{R} \approx-23$ (for $H_{0}=75 \mathrm{~km} \mathrm{~s}^{-1} \mathrm{Mpc}^{-1}$ and $q_{0}=0.0$ ). These galaxies would therefore have to be considerably more luminous than typical present-day galaxies (e.g., a present-day $L^{*}$ spiral has $M_{R} \approx-21$ ). In contrast, Bergeron \& Boisse (1991) have studied a sample of 13 galaxies at $z \approx 0.5$ identified with intervening $\mathrm{Mg}$ II absorption in high- $z$ quasars. They find that these galaxies have an average value of $M_{R} \approx-20.5$ (e.g., only $10 \%$ as luminous as our average case), with their most luminous example only having $M_{R} \approx-21.3$. It seems quite unlikely that the galaxies responsible for producing the $\mathrm{Mg}$ II absorption in our QSR sample would differ so dramatically and systematically from those in the Bergeron \& Boisse sample. Note that correcting the fuzz in our sample for the "missing" flux in the central most $\approx 2 \operatorname{arcsec}^{2}$ (see § 2) would strengthen this conclusion.

If the spatially resolved structures around the QSRs are intervening galaxies, in some cases they also have to be unusually blue compared to present-day galaxies. That is, the fuzz around Q0445+097 would be as blue as present-day Magellanic Irregular galaxies and the fuzz around Q1318+113 would be even bluer (see Fig. 4 and Table 2). Galaxies this blue must have extensive star formation and cor- 

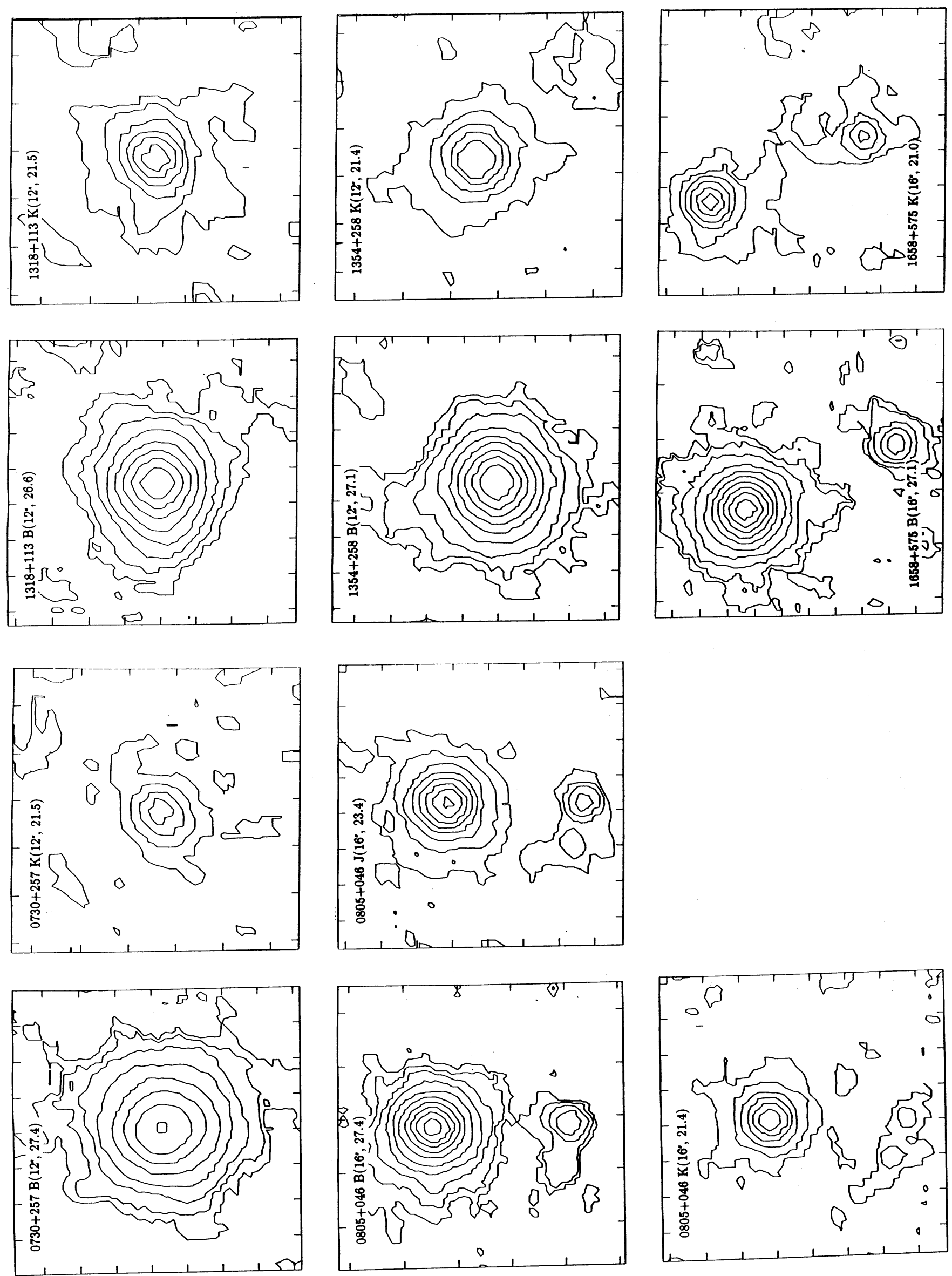
TABLE 2

Summary OF QSR “FuZz” ContinuUm Properties

\begin{tabular}{|c|c|c|c|c|c|c|c|c|c|c|}
\hline $\begin{array}{l}\text { QSR } \\
\text { (1) }\end{array}$ & $\begin{array}{l}\text { Filter } \\
\text { (2) }\end{array}$ & $\begin{array}{l}\text { Resolved/Total } \\
\text { (3) }\end{array}$ & $\begin{array}{c}m_{\text {tot }} \\
(4)\end{array}$ & $\begin{array}{l}m_{\text {resolved }} \\
\text { (5) }\end{array}$ & $\begin{array}{l}S_{v} \\
(6)\end{array}$ & $\begin{array}{l}F_{\text {em }} \\
(7)\end{array}$ & $\begin{array}{c}A \\
(8)\end{array}$ & $\begin{array}{l}M_{V} \\
(9)\end{array}$ & $\begin{array}{c}\alpha_{K}^{B} \text { (fuzz) } \\
(10)\end{array}$ & $\begin{array}{c}\alpha_{K}^{B} \text { (QSR) } \\
(11)\end{array}$ \\
\hline $\begin{array}{l}\mathrm{Q} 0445+097 \\
\quad(z=2.111) \ldots \ldots \ldots\end{array}$ & $\begin{array}{l}U \\
B \\
R \\
K\end{array}$ & $\begin{array}{l}0.38 \\
0.21 \\
0.21 \\
0.17\end{array}$ & $\begin{array}{l}20.2 \\
20.7 \\
19.2 \\
15.6\end{array}$ & $\begin{array}{l}21.3 \mathrm{a} \\
22.4 \mathrm{a} \\
20.9 \mathrm{a} \\
17.5 \mathrm{a}\end{array}$ & $\begin{array}{c}6.5 \\
4.7 \\
11 \\
69\end{array}$ & $\begin{array}{l}0.20 \\
0.08 \\
0.00 \\
0.03\end{array}$ & $\begin{array}{l}0.85 \\
0.72 \\
0.45 \\
0\end{array}$ & -25.2 & 1.4 & 1.4 \\
\hline $\begin{array}{l}\mathrm{Q} 0730+257 \\
\quad(z=2.686) \ldots \ldots . .\end{array}$ & $\begin{array}{l}B \\
K\end{array}$ & $\begin{array}{r}<0.04 \\
0.18\end{array}$ & $\begin{array}{l}19.6 \\
17.5\end{array}$ & $\begin{array}{l}>23.1 \\
19.4 \mathrm{~b}\end{array}$ & $\begin{array}{l}<2.5 \\
12\end{array}$ & $\begin{array}{r}>0.19 \\
0.00\end{array}$ & $\begin{array}{l}0.20 \\
0\end{array}$ & -24.2 & $>0.9$ & -0.1 \\
\hline $\begin{array}{l}\mathrm{Q} 0805+046 \\
\quad(z=2.877) \ldots \ldots \ldots\end{array}$ & $\begin{array}{l}B \\
J \\
K\end{array}$ & $\begin{array}{r}<0.04 \\
0.12 \\
0.14\end{array}$ & $\begin{array}{l}18.7 \\
16.6 \\
15.7\end{array}$ & $\begin{array}{r}>22.1 \\
18.9 \mathrm{a} \\
17.8 \mathrm{a}\end{array}$ & $\begin{array}{l}<5.6 \\
44 \\
47\end{array}$ & $\begin{array}{r}>0.85 \\
0.00 \\
0.00\end{array}$ & $\begin{array}{l}0 \\
0 \\
0\end{array}$ & -26.0 & $>1.3$ & 0.5 \\
\hline $\begin{array}{l}\mathrm{Q} 1318+113 \\
\quad(z=2.171) \ldots \ldots \ldots\end{array}$ & $\begin{array}{l}B \\
K\end{array}$ & $\begin{array}{l}0.16 \\
0.14\end{array}$ & $\begin{array}{l}19.1 \\
16.0\end{array}$ & $\begin{array}{l}21.1 \mathrm{a} \\
18.1 \mathrm{a}\end{array}$ & $\begin{array}{l}16 \\
36\end{array}$ & $\begin{array}{l}0.13 \\
0.13\end{array}$ & $\begin{array}{l}0 \\
0\end{array}$ & -24.8 & 0.5 & 0.5 \\
\hline $\begin{array}{l}\mathrm{Q} 1354+258 \\
\quad(z=2.032) \ldots \ldots \ldots\end{array}$ & $\begin{array}{l}B \\
K\end{array}$ & $\begin{array}{c}(0.03) \\
0.17\end{array}$ & $\begin{array}{l}18.9 \\
16.2\end{array}$ & $\begin{array}{l}(22.7 \mathrm{~b}) \\
18.1 \mathrm{a}\end{array}$ & $\begin{array}{l}(3.5) \\
36\end{array}$ & $\begin{array}{l}0.09 \\
0.01\end{array}$ & $\begin{array}{l}0 \\
0\end{array}$ & -24.4 & (1.4) & 0.3 \\
\hline $\begin{array}{l}\mathrm{Q} 1658+575 \\
\quad(z=2.173) \ldots \ldots \ldots\end{array}$ & $\begin{array}{l}B \\
K\end{array}$ & $\begin{array}{c}(0.06) \\
0.26\end{array}$ & $\begin{array}{l}18.4 \\
15.6\end{array}$ & $\begin{array}{c}(21.5 \mathrm{~b}) \\
17.1 \mathrm{a}\end{array}$ & $\begin{array}{c}(11) \\
96\end{array}$ & $\begin{array}{l}0.10 \\
0.02\end{array}$ & $\begin{array}{l}0 \\
0\end{array}$ & -25.7 & (1.3) & 0.3 \\
\hline
\end{tabular}

CoL. (3).- The fraction of the total flux within a $\approx 10^{\prime \prime} \times 10^{\prime \prime}$ box centered on the QSR that is spatially resolved (is in excess of the flux expected for a point source with the same flux as the QSR within the central-most $\approx 2 \operatorname{arcsec}^{2}$ of the image). Uncertainties are typically \pm 0.02 in these units. We regard all the QSRs to be resolved in the near-IR images, Q0445+097 and Q1318+113 to be resolved in the optical images, Q1354+258 and Q1658+575 to be possibly resolved in the optical images, and Q0730+257 and Q0805+046 to be unresolved in the optical images. See text for details.

CoL. (4).- The apparent magnitude of the QSR plus fuzz within a $10^{\prime \prime} \times 10^{\prime \prime}$ box centered on the QSR. The uncertainties are \pm 0.1 mag due to uncertainties in the photometric calibration.

CoL. (5).-The apparent magnitude of the spatially resolved "fuzz." Uncertainties are due to the uncertainties in resolved fractions (col. [3]), sky-noise, and uncertainties in the photometric calibration. The overall uncertainties are given by the letters following the magnitude, with " a" and " $b$ " denoting \pm 0.4 and \pm 0.8 mag, respectively.

CoL. (6). - The flux densities of the fuzz in microjanskys. The uncertainties are those given in the notes for col. (5), except at $U$ where the nonstandard effective bandpass defined by the $U$ filter, the fall-off of the TI CCD sensitivity at short wavelengths, and the uncertain effect of the Ly $\alpha$ forest on the fuzz SED makes a conversion from $U$ counts to a flux density particularly uncertain (we estimate \pm 0.3 dex). We have used the conversions from magnitudes to flux densities given in Allen 1976. The assumed flux densities for zero magnitudes at $U, B, R, J$, and $K$ were taken to be $2053,4264,2523,1600$, and $642 \mathrm{Jy}$, respectively.

CoL. (7). - The estimated fraction of the flux in the fuzz produced by emission-lines transmitted by the broad-band filters (see text for details).

Col. (8).- The extinction due to dust in the Milky Way in the bandpass of the filter given in col. (2.) The extinction is derived from the sky maps of color excess published by Burstein \& Heiles (1982) and the standard Milky Way extinction curve in Osterbrock (1989).

CoL. (9).- The absolute visual magnitude of the spatially resolved fuzz. These have been calculated by using the available fuzz flux densities to estimate a flux density at the observed wavelength corresponding to the redshifted center of the $V$ band: $\lambda=(1+z) 5470 \AA$. This was generally close to the center of the $K$-band, so interpolation uncertainties are small. The monochromatic power at a rest wavelength of $5470 \AA$ was then computed for our adopted cosmological parameters of $H_{0}=75 \mathrm{~km} \mathrm{~s}^{-1} \mathrm{Mpc}^{-1}$ and $q_{0}=0$ (note that for this redshift range the computed powers would be nearly the same for an $H_{0}=50 \mathrm{~km} \mathrm{~s}^{-1} \mathrm{Mpc}^{-1}$ and $q_{0}=0.5$ cosmology). This power was then converted to an absolute magnitude using a flux-density of $3830 \mathrm{Jy}$ for $V=0$ (Allen 1976). The derived values have been corrected for Milky Way extinction (col. [7]) but not for the presence of emission lines. We have not corrected these absolute magnitudes for the "missing" flux in the central-most $\approx 2$ arcsec $^{2}$, but estimate that this would brighten the fuzz by several tenths of a magnitude (by analogy to images of high- $z$ radio galaxies). The estimated uncertainties in $M_{V}$ are \pm 0.5 magnitudes except for Q0730 +259 ( $\left.\pm 0.8 \mathrm{mag}\right)$.

CoL. (10). - The effective spectral index between the $B$ and $K$ bands for the fuzz: $\alpha_{K}^{B} \equiv-\log \left(S_{v}^{B} / S_{v}^{K}\right) / \log \left(v_{B} / v_{K}\right)$. These values have been corrected for Milky Way extinction but not for the presence of emission lines (the latter would be $\leq 0.1$ in any case). The uncertainties are \pm 0.2 for $\mathrm{Q} 0445+097, \pm 0.3$ for $\mathrm{Q} 1318+113$, and \pm 0.5 for the other two cases.

CoL. (11). - Same as for col. (9), but for the unresolved component only (the QSR-proper). Uncertainties are \pm 0.1 .

respondingly strong nebular line emission. For example, Gallagher, Hunter, \& Bushouse (1989) find a mean rest-frame equivalent width for [O II] $\lambda 3727$ emission of $\approx 50 \AA$ in the present-day blue galaxies, corresponding to a measured equivalent width of $\approx 100 \AA$ at $z=1$. Even accounting for severe dilution of the fuzz light by the central QSR, the predicted equivalent width would still be 5-20 $\AA$. In contrast, the QSR spectra in Barthel, Tytler, \& Thompson (1990) and Heckman et al. (1991b) lead to upper limits of only $<0.5 \AA$ for any such emission lines. The details of this comparison can be found in Table 3. Thus if the structures around the QSRs are fore- ground galaxies, in some cases the objects must have line emission that is at least 10-40 times smaller (per unit starlight) than present-day galaxies with similar integrated colors.

A final argument against interpreting the QSR fuzz as due to intervening galaxies is its location along the $K$ versus $z$ Hubble diagram for radio galaxies. This must be a very unlikely coincidence if these structures are unrelated foreground systems (why would an intervening absorber "know" what apparent $K$ magnitude to have?).

It has been suggested that much of the continuum emission associated with high-redshift radio galaxies is not starlight at 


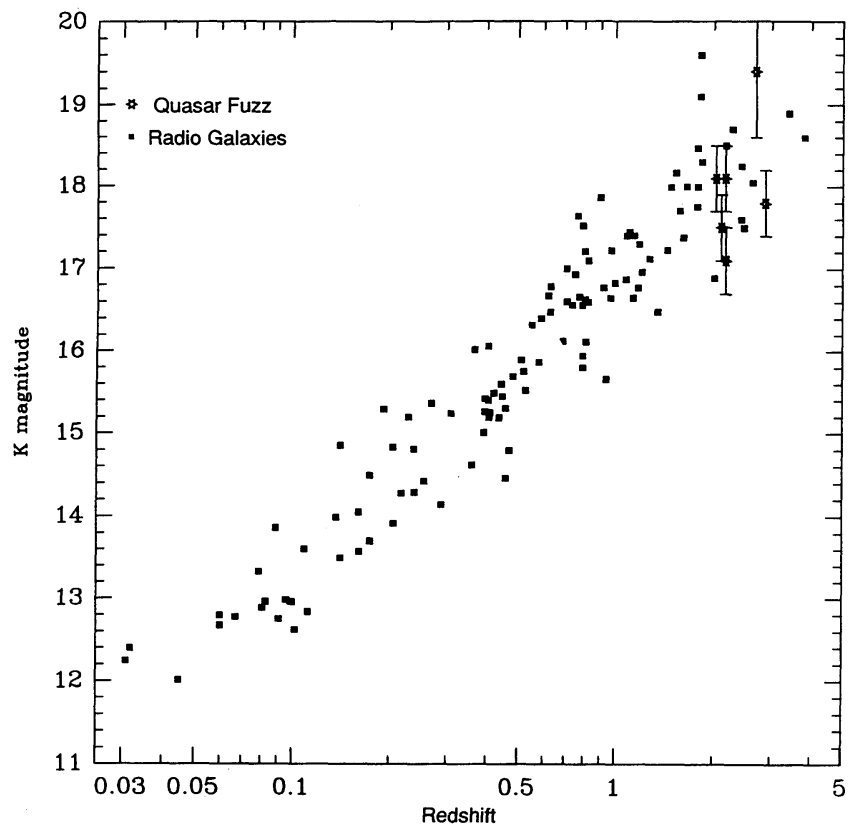

FIG. 3.-The six QSRs in our sample plotted on the "IR Hubble Diagram" (apparent K magnitude vs. redshift) for radio galaxies (Lilly 1989; Chokshi \& Eisenhardt 1991, McCarthy, Persson, \& West 1992). The QSRs overlap with the high-redshift radio galaxies in this plot. See Table 2 for details.

TABLE 3

Intervening Mg in Systems and Implied Fuzz Properties

\begin{tabular}{ccccr}
\hline \hline $\begin{array}{c}\text { QSR } \\
(1)\end{array}$ & $\begin{array}{c}z_{\text {abs }} \\
(2)\end{array}$ & $\begin{array}{c}M_{R} \\
(3)\end{array}$ & $\begin{array}{c}\text { Flux }_{\text {[OII] }} \\
(4)\end{array}$ & \multicolumn{1}{c}{$\begin{array}{c}\mathrm{EQW}_{\text {[OII] }} \\
(5)\end{array}$} \\
\hline $\mathrm{Q} 0445+097 \ldots \ldots \ldots$ & 0.84 & -23.3 & $6.6(\mathrm{E}-16)$ & $19(<0.5)$ \\
$\mathrm{Q} 0805+046 \ldots \ldots \ldots$ & 0.70 & -22.9 & $7.0(\mathrm{E}-16)$ & $5(<0.5)$ \\
$\mathrm{Q} 1318+113 \ldots \ldots \ldots$ & 0.84 & -22.8 & $1.2(\mathrm{E}-15)$ & $14(<0.5)$ \\
& 1.05 & -23.4 & $1.1(\mathrm{E}-15)$ & $15(<1.0)$ \\
$\mathrm{Q} 1354+258 \ldots \ldots \ldots$ & 0.86 & -22.3 & $4.1(\mathrm{E}-16)$ & $5(<0.5)$ \\
& 0.89 & -22.4 & $4.1(\mathrm{E}-16)$ & $5(<0.5)$ \\
& 1.42 & -23.0 & $5.7(\mathrm{E}-16)$ & 10 \\
\hline
\end{tabular}

CoL. (2).- Redshift of the $\mathrm{Mg}$ II $\lambda 2798$ doublet absorption-line systems taken from Barthel, Tytler, \& Thompson 1990.

CoL. (3).- The implied absolute $R$ magnitude of the QSR fuzz (properties given in Table 2) if it is at the redshift of the $\mathrm{Mg}$ II absorber. The procedure followed for computing $M_{R}$ was exactly analogous to that used for $M_{V}$ as described in the footnotes for Table 2 (Col. [8]). The uncertainties are $\pm 0.5 \mathrm{mag}$. We have not corrected these absolute magnitudes for the "missing" flux in the central-most $\approx 2 \operatorname{arcsec}^{2}$, but estimate that this would brighten the fuzz by several tenths of a magnitude (by analogy to images of high- $z$ radio galaxies).

Col. (4). - The predicted flux of the [O $\mathrm{I}] \lambda 3727$ emission-line (ergs $\mathrm{cm}^{-2} \mathrm{~s}^{-1}$ ) if the QSR fuzz is identified with $\mathrm{Mg}$ II absorber and if the foreground galaxy has [O II] emission that is as strong (rest equivalent width $\approx 50 \AA$ ) as present-day galaxies with similar spectral energy distributions as the QSR fuzz (see text for details).

CoL. (5). - The predicted equivalent width of [O II] $\lambda 3727$ emission (in angstroms in the observer's frame) from the intervening galaxy with respect to the total QSR light. The equivalent width is derived from the flux in col. (4) and the interpolated total QSR flux at the appropriate wavelength (see Table 2). The values given in parentheses are the estimated upper limits to [O II] equivalent widths, using the spectra in Barthel, Tytler, \& Thompson (1990). No data exist at the wavelength of the predicted [O II] emission in the $z=1.42$ absorption system in Q1354+258. all, but is instead light from a powerful AGN that is hidden from direct view along our line of sight (e.g., Fabian 1989). Radiation from this AGN is hypothesized to escape anisotropically along the radio source axis where it is scattered into our line of sight by dust or electrons in the galaxy halo, by analogy to the process that occurs in some Seyfert galaxies (e.g., Antonucci \& Miller 1985). This idea is supported by the data of di Serego Alighieri et al. (1989) and Scarrott, Rolph, \& Tadhunter (1990), who find that the spatially extended optical continuum emission in $3 \mathrm{C} 368$ is significantly polarized.

This model predicts that the scattered light should be similar in color to the AGN in the event of electron scattering and bluer than the central AGN in the event of scattering by normal dust grains. Since the color of the hidden AGN in high- $z$ radio galaxies is not known a priori, such a color test is impractical. However, we can straightforwardly make this test for our QSRs and their fuzz. As noted in $\S 3$ above, the fuzz is much redder than the QSR $-\geq 1.5 \mathrm{mag}$ in $(B-K)$ for four of the six cases. In Q0445+097 and Q1318+113 the fuzz and QSR have similar colors. This strongly suggests that the bulk of the near-IR light in the majority of cases cannot be scattered QSR light. The existence of the tight $K$ versus $z$ Hubble diagram and the location of the QSR fuzz along it is an additional argument against scattering being important at $K$ in either the high- $z$ radio galaxies or QSRs (see also Lilly 1990 and Chambers \& Miley 1990).

It is more difficult to discount the possibility that scattered QSR light makes an important contribution to the fuzz in the optical (rest-frame UV) images. That is, a two-component model in which the near-IR fuzz represents light from an old stellar population of the QSR host galaxy and the optical fuzz is scattered QSR light cannot be disproven. However, neither

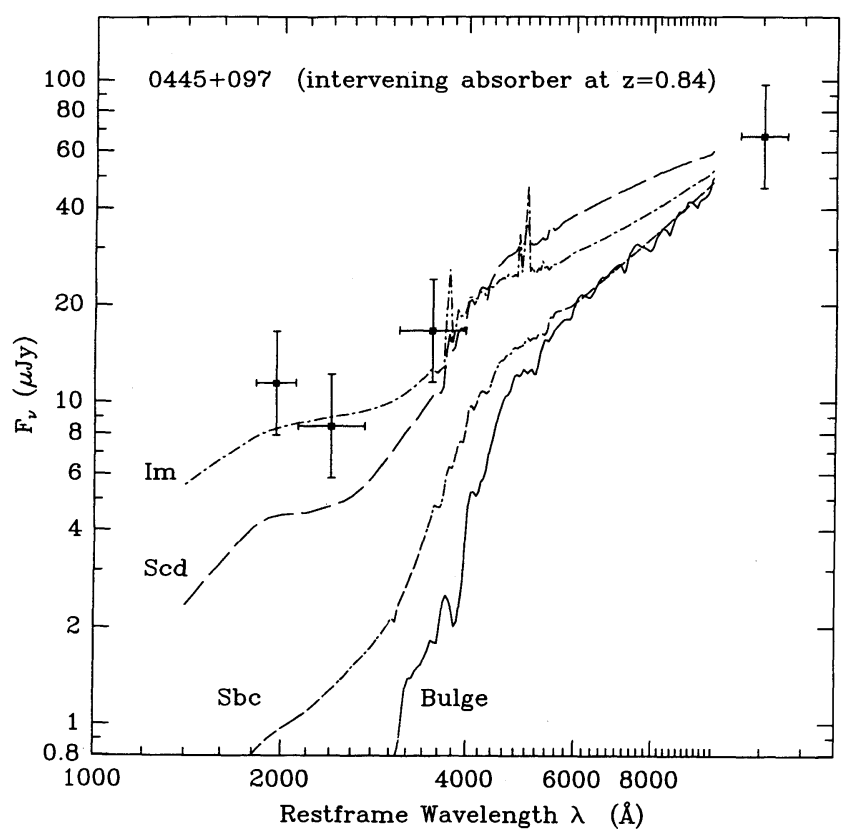

Fig. 4. - The spectral energy distribution for the fuzz around Q0445+097 (corrected for excitation due to Milky Way dust and for the presence of emission-lines), shifted to the rest-frame of the $\mathrm{Mg}$ II $\lambda 2798$ absorption system at $z=0.84$, and then overlaid on the spectral energy distributions of presentday galaxies of different Hubble types (from CWW). If the QSR fuzz is produced by a galaxy at $z=0.84$, it must be about as blue as a present-day Magellanic Irregular or Markarian starburst galaxy. 
the strong morphological similarity between the $K$-band and the optical fuzz noted in $\S 3$ above nor the clear morphological differences between the spatially extended Ly $\alpha$ nebulosity and optical fuzz discussed by HLVM are naturally accounted for in such a model. If both the Ly $\alpha$ nebulae and optical continuum fuzz are ultimately produced by reprocessing of QSR light by interstellar matter, why would the optical continuum fuzz trace the old stellar population (the $K$-band fuzz) better than it traces the ionized interstellar gas (the Ly $\alpha$ fuzz)?

The detection of the $4000 \AA$ break in the rest-frame SED of the QSR fuzz would provide direct evidence that the fuzz light is produced by stars (e.g., Chambers \& Charlot 1990). Accordingly, we obtained images of the fuzz around Q0805 + 046 in both $J\left(\lambda_{\text {rest }}=3100 \AA\right)$ and $K\left(\lambda_{\text {rest }}=5700 \AA\right)$, straddling the $4000 \AA$ break. Unfortunately, the uncertainties in the fuzz fluxes are so large $(\approx 0.4 \mathrm{mag}$ at both $J$ and $K)$ that the lack of a detection of the $4000 \AA$ break is not significant. We measure $\log \left(S_{v}^{K} / S_{v}^{J}\right)=0.03 \pm 0.23$ for the fuzz, so that a break of a factor of $\approx 2$ is consistent with the data. Of course, the lack of a detectable break is also consistent with a nonstellar origin for the fuzz in this case.

\subsection{Stellar Population of the Fuzz}

In light of the discussion in the preceding section, probably the most natural interpretation of the continuum fuzz is that it is predominantly starlight arising in the host galaxy of the QSR. A comparison of the fuzz properties to those of galaxies in the present-day universe is particularly instructive. In making this comparison, we note that at these redshifts the implied linear sizes and luminosities are similar for either our adopted cosmology of $H_{0}=75 \mathrm{~km} \mathrm{~s}^{-1} \mathrm{Mpc}^{-1}$ and $q_{0}=0.0$ or $H_{0}=50 \mathrm{~km} \mathrm{~s}^{-1} \mathrm{Mpc}^{-1}$ and $q_{0}=0.5$.

As discussed in more detail in $\S 3$, the typical isophotal sizes of the fuzz are $6 "-10^{\prime \prime}(\approx 50-90 \mathrm{kpc})$. Even more impressive than these large dimensions are the fuzz luminosities. Using the $K$-band apparent magnitudes we have calculated the absolute visual magnitudes for the fuzz. Since the $K$-band images typically sample rest wavelengths of $6000-7000 \AA$, calculations of $M_{V}$ involve only a modest interpolation in wavelength (which we made using the measured fuzz colors). The results (see Table 2) are values for $M_{V}$ ranging from -24.2 to -26.0 with a mean value of -25.1 . If the photometric structure of the QSR fuzz is similar to that of the high- $z$ radio galaxies, the "missing" light from the central-most $\approx 2 \operatorname{arcsec}^{2}$ (see $\S 2$ ) would make the QSR host galaxies several tenths of a magnitude more luminous still. To put this into perspective, the most luminous class of galaxies in the present universe (the first-ranked giant ellipticals in rich clusters) have $\left\langle M_{V}\right\rangle=-22.4 \pm 0.3$ (e.g., Sandage 1972 ) and the most luminous of the $\approx 1250$ galaxies in the Revised Shapely-Ames Catalog (Sandage \& Tammann 1987) has $M_{V}=-23.4$ (excluding any correction for internal absorption in the galaxy, since we have made no such correction in the case of the QSR fuzz).

We have parameterized the fuzz colors between approximate rest-frame wavelengths of 1300 and $6500 \AA$ by $\alpha_{K}^{B}$, the effective spectral index between $B$ and $K$ (see $\S 3$ and Table 2). The fuzz around $Q 0455+097$ has $\alpha_{K}^{B}=1.4$, but the fuzz around $\mathrm{Q} 1318+113$ is much bluer $\left(\alpha_{K}^{B}=0.5\right)$. For comparison, the SEDs of local galaxies given in Coleman, $\mathrm{Wu}, \&$ Weedman (1980, hereafter CWW) correspond to equivalent $\alpha$ 's of 1.1 for Magellanic Irregulars and 1.8 for Scd galaxies. This can be seen in more detail in Figure 5, where we display the measured color for the fuzz around Q0445 + 097 (the best-resolved case) super-

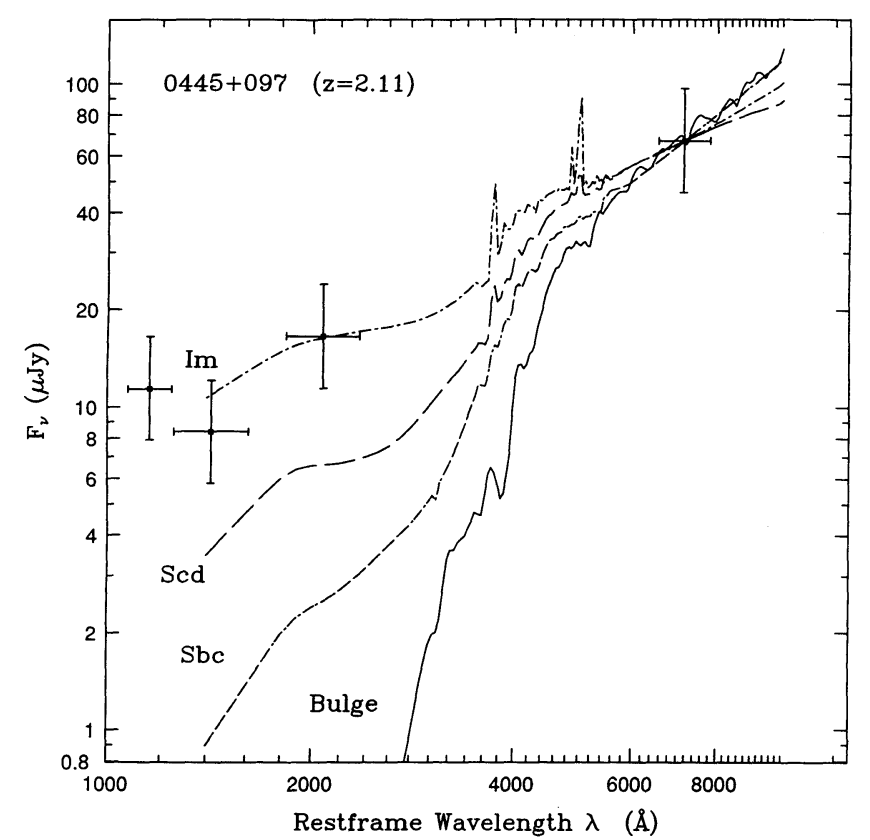

FIG. 5.-The spectral energy distribution for the fuzz around $\mathrm{Q} 0445+097$ (corrected for Milky Way extinction and QSR-fuzz emission-lines) shifted to the rest frame of the QSR and overlaid on the spectral energy distribution of present-day galaxies of different Hubble types (from CWW). The QSR fuzz is about as blue as the most actively star-forming classes of present-day galaxies (Magellanic Irregular and Markarian starburst galaxies).

posed on the SEDs of local galaxies of different Hubble types. The only galaxies in the local universe with colors are blue as the $\mathrm{Q} 1318+113$ fuzz are some members of the Markarian starburst population. We have used IUE fluxes at $1400 \AA$ reported in Weedman (1988) and $R$-band magnitudes measured by Huchra (1977) through apertures similar in size to the $10^{\prime \prime} \times 15^{\prime \prime}$ IUE aperture to derive a spectral index between 1400 and $6500 \AA$ for nine Markarian starburst galaxies. We find a mean value of $\alpha \approx 1.0$ (similar to the Magellanic Irregular SED in CWW), but colors as blue as $\alpha \approx-0.2$ (Mrk 59) and $\alpha \approx 0.1$ (Mrk 153) exist. Of course, the Markarian starburst galaxies are $\approx 300$ times less luminous than the QSR fuzz $\left(\left\langle M_{V}\right\rangle \approx-19\right.$ for the nine starbursts vs. $\approx-25$ for the QSR fuzz)!

Needless to say, the actual star-formation histories of the QSR fuzz cannot be determined in detail based upon the present meager data. Nevertheless, it is instructive to compare the properties of the fuzz around Q0445+097 and Q1318 + 113 (the two best-resolved cases) to both local starforming galaxies and to models made for the SEDs of highredshift radio galaxies. These comparisons enable us to make educated guesses about the possible star-formation histories of the QSR fuzz.

Present-day Magellanic Irregular galaxies have $\mathbf{H} \alpha$ emission-line equivalent widths of 25-100 $\AA$ (e.g., Gavazzi, Boselli, \& Kennicutt 1991), implying a star-formation rate of $\approx 1 M_{\odot}$ per year for a typical Irr with $M_{V} \approx-19$ (e.g., Kennicutt 1983). We have shown above that the QSR fuzz for Q0445 + 097 and Q1318 + 113 have SEDs similar to an Irregular. The star-formation rates in the fuzz implied by simply scaling up to the fuzz luminosities of $M_{V} \approx-25$ are then a few hundred $M_{\odot}$ per year. These values agree with the estimates made by HLVM on the basis of the $\approx 1300 \AA$ continuum luminosity alone. 
The SED of the fuzz can also be compared to models made for high-z radio galaxies. Chambers \& Charlot (1990) have published models made by assuming star-formation with a Miller-Scalo IMF and a rate that is declining exponentially with an $e$-folding time of 50 million years. We find that a reasonable fit to the SED of Q0445 + 097 can be made if the time since the peak in the star-formation rate is $\approx 200$ million years. The stellar population is therefore best categorized as "poststarburst." We note that Lilly (1989) achieves equally good fits to similar data with a combination of an old (> gigayear) population providing most of the mass and $K$-band light and a starburst population (ongoing starformation rate of several hundred $M_{\odot}$ per year) that provides the optical (rest-frame UV) light.

\subsection{Whither the High-z QSR Fuzz?}

The properties of the QSR fuzz are so extreme that is interesting to consider what such objects would evolve into between $z=2$ to 3 and $z \approx 0$. Applying the old-population-plus-burst models made by Lilly $(1989,1990)$ for high $-z$ radio galaxies to the QSR hosts imply that they will fade by $\approx 2.5-3$ mag at rest-frame $V$, while "postburst" models of Chambers \& Charlot (1990) imply fading by 3-4 mag at rest frame $V$ for ages of 200 to 300 million years for the QSR hosts (see also Charlot \& Bruzual 1991). Both types of models therefore predict that the QSR hosts will evolve to present-day galaxies with $M_{V} \approx-22$, comparable to present-day first-ranked cluster giant ellipticals.

The present space density of first-ranked cluster galaxies - as given by the space density of Abell richness class $\geq 0$ clusters derived by Batuski et al. (1991)-is $\approx 5000 \mathrm{Gpc}^{-3}$ (for our choice of $H_{0}=75 \mathrm{~km} \mathrm{~s}^{-1} \mathrm{Mpc}^{-1}$ and $q_{0}=0$ ). Not all such luminous galaxies are first-ranked cluster galaxies, however. The field galaxy luminosity function determined by Efstathiou, Ellis, \& Peterson (1988) yields a space density of $\approx 5 \times 10^{4}$ $\mathrm{Gpc}^{-3} \mathrm{mag}^{-1}$ for galaxies with $L \approx L_{\mathrm{FRCG}} \approx 3.6 L^{*}$. In comparison, the co-moving space density of all quasars with $M_{B}$ brighter than -25 at $z \approx 2.5$ is $\approx 2000 \mathrm{Gpc}^{-3}$ (see Hartwick \& Schade 1990), while the corresponding space density of radio sources as powerful as our QSRs is only $\sim 3 \mathrm{Gpc}^{-3}$ (Dunlop \& Peacock 1990). The space density of the sites of the resulting dead quasars would be greater than these values by a factor given approximately by the ratio of the duration of the "quasar epoch" at high redshifts (one-to-several gigayears) to the lifetime at this luminosity of an average high- $z$ quasar.

We conclude that a shortage of available sites for dead quasars in the present universe develops only if high- $z$ quasars have short lifetimes ( $<$ few times $10^{8} \mathrm{yr}$ ) and if most high- $z$ quasars (not just the very rare radio-loud ones we have investigated) are immersed in fuzz with $M_{V} \approx-25$. The latter can be directly tested by $K$-band imaging of high- $z$ radio-quiet quasars spanning a broad range in absolute magnitude.

\subsection{Relationship to Low-Redshift QSR Fuzz}

It is suggestive that the absolute magnitudes of the host galaxies of low redshift $(z \approx 0.3)$ QSRs are similar to those predicted by the models for the "faded" fuzz of the high-z QSRs (e.g., Smith et al. 1986 find $\left\langle M_{V}\right\rangle=-22.4$ for the fuzz around 30 low-z QSRs; see also Hutchings 1987). While this might suggest that individual high- $z$ QSRs evolve directly into low- $z$ QSRs, this result is hard to reconcile with the apparent evolution of the clustering environment around QSRs with redshift (e.g., Ellingson, Yee, \& Green 1991). More likely, it simply means that QSRs at any epoch tend to occur in the most massive galaxies. Lilly (1990) has made a similar argument concerning radio galaxies based on the $K$ versus $z$ diagram.

Whether or not the high-z QSRs evolve to low-z QSRs, the difference of a factor of 10 in the typical optical luminosity of the host galaxy between high- and low-redshift is quite striking. Relating this difference to the processes responsible for the strong cosmic evolution of the QSR population is a speculative enterprise at best. Imaging of lower luminosity QSRs at high-z would be valuable in this regard to determine whether the fuzz properties are primarily a function of luminosity or redshift. It will also be important to determine whether the QSR fuzz luminosity evolves more strongly with redshift than the general field galaxy population (see Cowie \& Lilly 1990; Koo 1990). Recent observations of apparent companion galaxies associated with QSRs suggest that this particular galaxy population has brightened by more than a magnitude out to redshifts of-order unity (Ellingson, Yee, \& Green 1991; Hintzen, Romanishin, \& Valdes 1991).

\section{SUMMARY AND CONCLUSIONS}

We have presented and discussed deep multicolor optical and near-irifrared images of six high-redshift $(z=2-3)$ radioloud quasars ("QSRs"). The QSRs are all well-resolved spatially in the near-IR ( $K$-band) images and are also resolved in at least two, and possibly four of six cases in the optical images. The structures surrounding the central point-source (QSR proper) provide less than $4 \%-40 \%$ of the total flux, have typical angular sizes of $\approx 5^{\prime \prime}-10^{\prime \prime}$, and apparent magnitudes ranging from 17 to 19 at $\mathrm{K}$ and 21 to $>23$ in the optical. The fuzz is significantly redder than the central QSR in the four of the six cases by at least $1.5 \mathrm{mag}(B-K)$. Parameterizing the spectral energy distributions by a spectral index between $B$ and $K(\approx 1300 \AA$ and $6500 \AA$ in the QSR rest frame) yields typical values of $\alpha_{K}^{B}=0.0-0.5$ for the central QSR and $\alpha_{K}^{B}=0.5-1.5$ for the best-resolved fuzz $\left(S_{v} \approx v^{-\alpha}\right)$. The amount of morphological information about the fuzz is quite limited, but the fuzz is usually asymmetric, and there is a marked morphological similarity between the optical and near-IR images.

The optical and near-IR properties of the QSR fuzz are strikingly similar to those of radio galaxies at similarly high redshifts. We find that the fuzz lies approximately along the tight $K$ magnitude versus redshift Hubble Diagram for radio galaxies (Lilly 1989). The spectral energy distributions we measure for the well-resolved cases are similar to those of the bluer high-redshift radio galaxies. There is suggestive evidence that the QSR fuzz may be preferentially aligned with the radio source axis (as is the case for radio galaxies), but this must be confirmed by imaging of a much larger sample. Clearly, QSRs and radio galaxies at high redshifts are closely related phenomena. This is consistent with, but does not require or prove, the idea that the two AGN classes are fundamentally the same population of objects viewed either along (QSR) or perpendicular to (radio galaxies) the radio axis (Barthel 1989).

We have considered three possible explanations for the origin of the QSR fuzz: light from the foreground galaxies responsible for producing the intervening $\mathbf{M g}$ II $\lambda 2798$ absorption-lines (e.g., LeFevre \& Hammer 1988), light from the central QSR scattered by dust or electrons in the halo of the QSR (e.g., Fabian 1989), and light from a population of stars in the host galaxy of the QSR (e.g., Chambers \& Charlot 1990; Lilly 1989; Chambers \& McCarthy 1990). We find that the 
galaxies responsible for the $\mathrm{Mg}$ II absorption are unlikely to make a significant contribution to the fuzz light unless they are systematically over 10 times more luminous than the typical Mg II absorbers identified by Bergeron \& Boisse (1991) and unless they are deficient in [O II] $\lambda 3727$ emission by more than an order-of-magnitude compared to present-day galaxies with similar colors. It would also be difficult to understand the location of the fuzz on the radio galaxy $K$ vs. $z$ diagram if the $K$ light was produced by an intervening galaxy.

Scattered QSR light should be at least as blue as the QSR itself, while we find the opposite effect: in four of six cases the fuzz is at least 1.5 mag redder than the QSR in $(B-K)$ and has approximately the same color in the other two. This result, together with the fuzz placement on the radio galaxy $K$ versus $z$ diagram means that scattered QSR light probably does not make a dominant contribution to the $K$-band fuzz light in most cases. However, scattering by interstellar matter could be significant in the optical images (though the morphological similarity of the optical and $K$-band continuum images on the one hand, and the morphological differences between the optical continuum and $\operatorname{Ly} \alpha$ images on the other make this a somewhat unnatural model in our view).

We believe that the most natural interpretation is that the QSR fuzz is produced primarily by the stellar population of the host galaxy of the QSR. Comparison of the properties of these QSR galaxies to present-day galaxies is revealing. For $H_{0}=75$ $\mathrm{km} \mathrm{s}^{-1} \mathrm{Mpc}^{-1}$ and $q_{0}=0$, the luminosities of the QSR hosts range from $M_{V}=-24.2$ to -26.0 (mean -25.1 ). This is $\approx 12$ times more luminous than the most luminous class of present- day galaxies (the first-ranked cluster galaxies). Corrections for galaxy light in the central-most $\approx 2 \operatorname{arcsec}^{2}$ (which we do not directly measure) would make the high- $z$ QSR hosts several tenths of a magnitude more luminous still. The spectral energy distributions of the QSR fuzz in the best-resolved cases are similar to those of the most actively star-forming galaxies in the present-day universe (Magellanic Irregular and Markarian starburst galaxies). Scaling the star-formation rates for such local galaxies to the luminosities of the QSR fuzz implies starformation rates of several hundred solar masses per year, as expected for young high-mass galaxies.

By analogy to the models that have been made of the stellar populations of high-redshift radio galaxies, the QSR fuzz could fade to the absolute magnitude of a present-day giant elliptical by redshift zero. The present-day space density of galaxies this luminous is high enough to easily provide sites for "dead" high- $z$ QSRs unless all high- $z$ quasars (not just the rare radioloud ones) occur exclusively in such luminous galaxies. The hosts of high-z QSRs are $\sim 2.5-3$ mag more luminous onaverage in the rest-frame optical than the hosts of low- $z$ QSRs. We emphasize that the hosts of radio-loud quasars at any redshift are probably a subset of the most massive galaxies in existence. Lilly $(1989,1990)$ has reached similar conclusions for radio galaxies.

We would like to thank the staffs at CTIO, KPNO, and UKIRT for their help in obtaining the data presented in this paper.
Allen, C. 1976, Astrophysical Quantities (London: Athlone)

Antonucci, R., \& Miller, J. 1985, ApJ, 297, 621

Barthel, P. 1989, ApJ, 336, 606

Barthel, P., Miley, G., Schilizzi, R \& Lonsdale, C. 1988, A\&AS, 73, 515

Barthel, P., Tytler, D., \& Thomson, B. 1990, A\&AS, 82, 339

Batuski, D., Burns, J., Newberry, M., Hill, J., Deeg, H.-J., Laubscher, B., \& Elston, R. 1991, AJ, 101, 1983

Begelman, M., \& Cioffi, D. 1989, ApJ, 345, L21

Bergeron, J., \& Boisse, P. 1991, A\&A, 243, 344

Burstein, D., \& Heiles, C. 1982, AJ, 87, 1165

Carlberg, R. 1990, ApJ, 350, 505

Chambers, K., \& Charlot, S. 1990, ApJ, 348, L

Chambers, K., \& McCarthy, P. 1990, ApJ, 354, L9

Chambers, K., \& Miley, G. 1990, in Evolution of the Universe of Galaxies, ed. R. G. Kron (San Francisco: ASP) 373

Chambers, K., Miley, G., \& van Breugel, W. 1987, Nature, 329, 604

Charlot, S., \& Bruzual, G. 1991, ApJ, 367, 126

Chokshi, A., \& Eisenhardt, P. 1991, preprint

Coleman, G., Wu, C., \& Weedman, D. 1980, ApJS, 43, 393 (CWW)

Cowie, L., \& Lilly, S. 1990, in Evolution of the Universe of Galaxies, ed. R. G. Kron (San Francisco: ASP), 212

Crawford, C., Fabian, A., \& Johnstone, R. 1988, MNRAS, 235, 183

di Serego Alighieri, S., Fosbury, R., Quinn, P., \& Tadhunter, C. 1989, Nature, 341,307

Dunlop, J., \& Peacock, J. 1990, MNRAS, 247, 19

Efstathiou, G., Ellis, R., \& Peterson, B. 1988, MNRAS, 232, 431

Elias, J., Frogel, J., Mathews, K., \& Neugebauer, G. 1982, AJ, 87, 1029

Ellingson, E., Yee, H., \& Green, R. 1991, ApJ, 371, 49

Fabian, A. 1989, MNRAS, 238, 41P

Francis, P., Hewett, P., Foltz, C., Chaffee, F., Weymann, R., \& Morris, S. 1991, ApJ, 373, 465

Gallagher, J., Hunter, D., \& Bushouse, H. 1989, AJ, 97, 700

Gavazzi, G., Boselli, A., \& Kennicutt, R. 1991, AJ, 101, 1207

Graham, J. 1982, PASP, 94, 244

Hartwick, F., \& Schade, D. 1990, ARAA, 28, 437

Heckman, T., Lehnert, M., van Breugel, W., \& Miley, G. 1991a, ApJ, 390, 100 (HLVM)

\section{REFERENCES}

Heckman, T., Lehnert, M., Miley, G., \& van Breugel, W. 1991b, ApJ, 381, 373

Hintzen, P., Romanishin, W., \& Valdes, F. 1991, ApJ, 366, 7

Huchra, J. 1977, ApJS, 35, 171

Hutchings, J. 1987, ApJ, 320, 122

Kennicutt, R. 1983, ApJ, 272, 54

Koo, D. 1990, in Evolution of the Universe of Galaxies, ed. R. G. Kron (San Francisco: ASP), 268

Le Fevre, O., \& Hammer, F. 1988, ApJ, 333, L37

Lilly, S. 1989, ApJ, 340, 77

1990, in Evolution of the Universe of Galaxies, ed. R. G. Kron (San Francisco: ASP), 344

McCarthy, P. 1991, in Connections between AGNs and Starburst Galaxies, ed A. Filippenko (San Francisco: ASP), in press

McCarthy, P., Elston, R., \& Eisenhardt, P. 1992, ApJ, 387, L29

McCarthy, P., Persson, S., \& West, S. 1992, ApJ, 386, 52

McCarthy, P., van Breugel, W., Spinrad, H., \& Djorgovski, S. 1987, ApJ, 321, L29

McLean, I. 1988, in Infrared Astronomy with Arrays, ed. C. G. Wynn-Williams \& E. E. Becklin (Honolulu: Univ. of Hawaii), 180

Neff, S., \& Hutchings, J. 1990, AJ, 100, 1441

Norman, C., \& Miley, G. K. 1984, A\&A, 141, 85

Osterbrock, D. 1989, Astrophysics of Gaseous Nebulae and Active Galactic Nuclei (Mill Valley, CA: University Science Books).

Rees, M. 1988, MNRAS, 231, 91P

Sandage, A. 1972, ApJ, 178, 25

Sandage, A., \& Tammann, G. 1987, A Revised Shapely-Ames Catalog of Bright Galaxies (Washington, DC: Carnegie Institution of Washington)

Sanders, D., Phinney, E. S., Neugebauer, G., Soifer, B. T., \& Matthews, K. 1989, ApJ, 347, 29

Scarrott, M., Rolph, C., \& Tadhunter, C. 1990, MNRAS, 243, 5P

Smith, E., Heckman, T., Bothun, G. Romanishin, W., \& Balick, B. 1986, ApJ, 306, 64

Smith, E., \& Heckman, T. 1990, ApJ, 348, 38

Turner, E. 1991, AJ, 101, 5

Weedman, D. 1988, Astrophys. Lett. Comm., 27, 117

Windhorst, R., et al. 1991, ApJ, 380, 362 